\title{
The role of comprehensive nutritional care in cancer patients
}

\author{
Michał Jankowski1,2, Anna Qelaj', Stanisław Kłęk³, Dawid Murawa4 , Małgorzata Nartowicz' , \\ Zbigniew Patela, Dorota Mańkowska-Wierzbicka5 , Aleksandra Kapała6, Barbara Kuczyńska7, \\ Wojciech Zegarski ${ }^{1,2}$
}

\begin{abstract}
${ }^{1}$ Chair of Surgical Oncology, Ludwik Rydygier Collegium Medicum in Bydgoszcz, Nicolaus Copernicus University in Torun, Bydgoszcz, Poland
${ }^{2}$ Department of Surgical Oncology, Oncology Center - Prof. Franciszek Lukaszczyk Memorial, Bydgoszcz, Poland

${ }^{3}$ Surgical Oncology Clinic, M. Sklodowska-Curie National Research Institute of Oncology Krakow Branch, Krakow, Poland

${ }^{4}$ Department of Surgery and Oncology, University of Medical Science, Zielona Gora, Poland

${ }^{5}$ Department of Gastroenterology, Metabolic Diseases, Internal Medicine and Dietetics, Poznan University of Medical Sciences, Poznan, Poland

${ }^{6}$ Department of Clinical Nutrition, M. Sklodowska-Curie National Research Institute of Oncology, Warsaw, Poland

'Department of General, Endocrinological Surgery and Gastroenterological Oncology, Poznan University of Medical Sciences, Poznan, Poland
\end{abstract}

Cancer patients often have inappropriately low energy intake, exhibit an increased loss of muscle proteins and generalized inflammatory status. Nutritional support aims to reverse these processes. Covering energy requirements is necessary for safety of anti-cancer treatments: radiotherapy, chemotherapy, surgery. Nutritional support and nutritional status monitoring should be managed at every stage of the disease. Nutritional intervention is most important in malnourished patients. Comprehensive, individualized nutritional care improves the results of treatment in cancer patients. Nutritional therapy is essential in obtaining the best results from anti-cancer treatment; however, it will be effective, if should completely cover nutrient requirements.

Key words: nutrition, cancer therapy

\section{Covering energy requirements as an element of anti-cancer therapy}

Deficiencies in nutrition state are commonly observed in patients with diagnosed cancer. The state of the organism's nutrition is one of the most essential elements that determines the overall condition of the body.

Malnutrition is a state resulting from malabsorption or ingestion of nutrients followed by changes in body composition. It can lead to the impairment of broadly understood organism activities
- both physical and mental [1]. The causes of malnutrition include insufficient oral nutrition, increased loss of nutrients and increased energy expenditure; these can all be related to the development of cancer. For malignant cancer patients, a negative protein and energy balance can additionally be escalated by lower food consumption as a result of anorexia and/or impaired absorption [2-4].

The body mass index (BMI), i.e. a measure of relative body mass based on the weight and height of a patient is one of

\section{How to cite:}

Jankowski M, Qelaj A, Klek S, Murawa D, Nartowicz M, Patela Z, Mańkowska-Wierzbicka D, Kapała A, Kuczyńska B, Zegarski W. The role of comprehensive nutritional care in cancer patients. NOWOTWORY J Oncol 2021; 71: 158-161. 
the primary parameters used for the assessment of nutritional status. BMI was identified as a prognostic factor for many types of cancers [5], i.e. a weight loss by $>10 \%$ over six months and/or a BMI below $20 \mathrm{~kg} / \mathrm{m}^{2}$ are identified in $75 \%$ of patients with newly diagnosed malignant cancers of the head and neck area [3].

Cancer patients have inappropriately low energy intake, exhibit an increased loss of muscle proteins and generalized inflammatory status that enhance the intensity of catabolic reactions in the body [2]. In those patients, we can observe an increased level of basic metabolic rate (BMR) that frequently co-exists with body mass loss, and is particularly exacerbated in lung and pancreatic carcinoma patients. It seems that the total metabolic rate (TMR) level in advanced cancer patients is lower when compared to healthy persons, which is associated with a reduction of the patients' physical activity $[2,6,7]$.

Similarly to healthy persons, nutritional therapy conducted in cancer patients should include an assessment of the TMR level. Nevertheless, we should note the potential risk for over-and underestimation of calorie intake in overweight and malnourished patients, respectively. We also have to remember that nutritional therapy in cancer patients is only valid when a patient receives all their essential nutrients, especially a high protein intake of $1-1.5 \mathrm{~g} / \mathrm{kg}$ of body weight (BW) per day. The energy intake should amount to $25-30 \mathrm{kcal} / \mathrm{kg}$ BW/day [2, 4].

Despite contradictory reports on the role of immunonutrition, it is believed that it has a particular significance in the perioperative period in patients with upper digestive tract cancer [8]. The nutrition is preferably administered via the oral or enteral route [9]. The diet prepared for cancer patients should also include eicosapentaenoic acid (EPA), an essential substrate for cyclooxygenase that limits an inflammatory response in the patient's body $[2,10]$.

The nutritional intervention used during radiochemotherapy in head and neck cancer patients positively impacts their nutritional status and quality of life [11]. Moreover, it results in markedly lower body mass loss compared to patients who do not receive such support [12].

Regarding chemotherapy, treatment results depend on the stage of cancer as well as on the level of body mass loss and the patient's nutritional status. A lower body mass loss during chemotherapy is associated with better results of anticancer therapy and improved survival of patients - even those with inoperable and unresectable lesions. On the other hand, a higher body mass loss and lower BMl are linked with a higher risk of complications. A normal nutritional status increases the chances of completing systemic therapy at the scheduled time and doses. [13-15].

Nutritional intervention is particularly important in the context of prevention and delay of cancer cachexia development. Cachexia is most often defined as a body mass loss $>5 \%$ in the previous six months or more than $2 \%$ when the patient's $\mathrm{BMl}$ is $<20 \mathrm{~kg} / \mathrm{m}^{2}$. Cachexia is defined as a multi-factor syndrome characterized by a permanent loss of skeletal muscle mass with a loss of body fat (or without it), which cannot be fully reversed by conventional nutritional support [16]. According to Fearon, cachexia is a multifactorial, dynamic and progressing process divided into 3 phases: pre-cachexia, cachexia and the final one: treatment-resistant cachexia (refractory cachexia) with an expected survival time below 3 months [17].

The pathophysiology of the syndrome is characterized by a negative protein and energy balance, affecting the overall quality of life in a negative way, reducing tolerance to treatment and decreasing the overall survival of cancer patients. In cancer patients, cachexia syndrome is one of the major causes of morbidity and mortality. The progressing cachexia indicates a poor prognosis with a shorter survival time and it accompanies nearly $20 \%$ of all deaths caused by cancer [18].

Nevertheless, it should be remembered that cancer patients with concurrent metabolic disorders and body mass loss receiving hypercaloric nutrition often do not gain bodyweight. Therefore, such an intervention is not recommended.

Malnutrition and muscle atrophy are often observed in cancer patients; they have a negative impact on the result of clinical treatment and lead to prolonged hospitalization. The most frequent causes include an increase in energy and protein demand resulting from the catabolic and physiological effects of cancer cachexia, inadequate dietary intake and decreased physical activity [19].

The fundamental nutritional problem experienced by patients suffering from cancer - and likely the one with the greatest influence on prognosis - is muscle atrophy (sarcopenia). A low muscle mass is common regardless of the stage of cancer (curative to palliative) and is an independent predictor of poor physical function, lower quality of life, surgical complications, cancer progression and decreased chances of survival [20].

Nutritional intervention enables the prevention and treatment of anti-cancer therapy-related complications, including surgery, as well as improves its efficacy and extends patients' survival.

In order to face the deterioration of the nutritional state, it is of great significance to collect data on nutritional status and its evolution over the course of the disease. Various types and sites of cancer present distinct nutritional models which require adjusted nutritional therapy. The deterioration of the state of nutrition is multifactorial - it can be a result directly related to cancer, nutrition and/or metabolism [21]. Nutritional interventions will differ depending on the medical history of patient, type and stage of cancer and response to therapy. If a patient can eat and has an efficient digestive tract, nutritional counseling with or without ONS should be the selected intervention in order to take account of the amended nutritional requirements caused by treatment or disease $[21,22]$. All cancer patients should be regularly controlled in terms of the development risk or presence of malnutrition. In all patients except for those provided with end of life care, it is necessary to meet all requirements regarding energy and 
protein to order nutritional interventions in a gradual way starting from counseling to parenteral nutrition. Nevertheless, the benefits and risk of nutritional interventions need to be well-balanced with particular reference to patients with advanced disease [22].

\section{Pharmacoeconomic aspects of nutritional therapy in cancer patients}

Anti-cancer treatment initiation in emaciated patients is associated with a mortality and infectious complications rate of $30 \%$ and $75 \%$, respectively. It should be remembered that a loss of lean body mass (LBM) and a reduction in physical capacity are signs accompanying both chemotherapy as well as advanced cancer. Body mass loss during chemotherapy has a direct impact on the therapeutic effect, among others resulting in a reduction of chemotherapy duration and the need to decrease doses of cytotoxic drugs. Moreover, it is associated with a decrease of the overall survival rate, the duration of the treatment response, a deterioration of patients' quality of life and performance status as well as with the worsening of general health conditions $[15,24,25]$. Comprehensive nutritional care should be proposed to every patient aimed at not only extending survival but also improving the quality of life. Nutritional support should be individualized and adjusted to the cancer stage.

In the preoperative preparation of patients at high nutritional risk, nutritional therapy is particularly important [26]. Oral nutritional supplements (ONS) facilitate an increase in nutrient intake and thus allows for stabilization or improvement in nutritional status. The patient should receive an appropriate intake which in case of immunonutrition consists of $3 \times 250 \mathrm{ml}$ of products containing arginine, n-3 fatty acids, or nucleotides [4]. Such supplementation is not reimbursed by the National Health Fund (NFZ) in Poland, even though it brings measurable benefits such as an improvement in the general health condition and also shortens the duration of the hospital stay [4, 9, 8]. It was demonstrated that the use of ONS as an intervention aiming only to improve nutrient intake resulted in a reduction of the re-hospitalization rate by $27.1 \%$ [27]. However, it is believed that the addition of immunomodulatory substances may boost that effect.

The pharmacoeconomic aspects of nutritional therapy in cancer patients illustrate the fact that two out of three surgical patients demonstrate nutritional status disorders. These disturbances are linked to a three fold increase in the risk of complications and a five fold increase in the risk of death compared to normally nourished persons. Unfortunately, despite the data mentioned above indicating the role of nutrition in cancer, only three out of four surgeons believe that peri-operative nutrition has a real impact on the number of complications. Moreover, only a small number of patients receive preoperative nutritional intervention despite the availability of sufficient evidence supporting the fact that the financing of nutrition therapy during the hospital stay results in the reduction of total treatment costs. This is confirmed by data showing that, on average, malnourished patients stay at the hospital for 17.2 days while patients without malnutrition only 9.7 days [28].

Elements of nutritional care are included and regulated by the comprehensive perioperative care protocol ERAS. Its use results in the average shortening of patients' hospital stay by 2.5 days, a reduction in the risk of complications by $40 \%$, non-surgical complications by $60 \%$ and respiratory and cardiovascular complications by $60 \%$ and $50 \%$ respectively [29]. Furthermore, it markedly increases the rate of 5-year survival [30]. Regardless of the amount of data indicating the importance of the ERAS protocol, its recommendations are implemented in only $1 \%$ of patients. Should this data not be sufficiently convincing, it is worth adding that the profitability of enteral, parenteral nutrition and PEG amounts to $>40 \%$, $>30 \%$, and $30-40 \%$ respectively [29].

\section{Discussion}

The above-mentioned considerations provoke discussions concerning several topics. The first topic concerns the patient's access to information. Emphasis was placed on the need to provide cancer patients with complete and understandable information about the desired mode of nutrition. Such information should also describe the possibility of using ONS to improve the patient's nutritional status. It is also essential to present this information to the patient at the moment of diagnosis in order to create the possibility of implementing nutritional therapy early on as a part of the comprehensive anti-cancer treatment $[2,28,31]$.

Particular attention was also paid to the fact that the frequency of patients referred to a nutritional consultation is increasing. However, those referrals are often delayed and in the majority of cases take place when anti-cancer treatment related complications emerge (for example, when chemotherapy cessation or surgery deferral is needed). The fact that hospitals only employ a small number of dietitians and that the estimated duration of a dietetic consultation, including the measurement of lean body mass (LBM) and a nutritional interview, is $30 \mathrm{mi}-$ nutes is vital as well. Taking into account the benefits of using nutritional support, we should also consider of funds relocation and enabling the patient to access this type of services.

No published randomized trials evaluate the role of nutritional care in cancer patients. However, a report by Schuetz demonstrates that the use of an appropriate, ESPEN guidelines-compliant model of nutritional care results in a reduction of complication rates and 30-day mortality rate of inpatient non-surgical patients compared to a control group receiving standard care [32].

\section{Conclusions}

Nutritional support and nutritional status monitoring should be managed at every stage of cancer. Comprehensive, indivi- 
dualized nutritional care improves the results of treatment in cancer patients. Nutritional intervention is most important in malnourished patients. Nutritional therapy is essential to obtain the best results from anti-cancer treatment; however, it should completely cover all nutrient requirements.

Conflict of interest: none declared

\section{Michał Jankowski}

Nicolaus Copernicus University in Torun

Ludwik Rydygier Collegium Medicum in Bydgoszcz

Chair of Surgical Oncology

ul. Romanowskiej 2

85-796 Bydgoszcz, Poland

e-mail:michaljankowski@post.pl

\section{Received: 24 Oct 2020}

Accepted: 19 Jan 2021

\section{References}

1. Sobotka L. Basics in clinical nutrition. 4th ed. Galen 2012.

2. Arends J, Bachmann P, Baracos V, et al. ESPEN guidelines on nutrition in cancer patients. Clin Nutr. 2017; 36(1): 11-48, doi: 10.1016/j. clnu.2016.07.015, indexed in Pubmed: 27637832.

3. Kapała A. Nutritional therapy during the treatment of head and neck cancer. Oncol Clin Pract. 2018; 14(2): 79-85, doi: 10.5603/ OCP.2018.0012 .

4. Weimann A, Braga M, Carli F, et al. ESPEN guideline: Clinical nutrition in surgery. Clin Nutr. 2017; 36(3): 623-650, doi: 10.1016/j.clnu.2017.02.013, indexed in Pubmed: 28385477.

5. Li ZQ, Zou L, Liu TR, et al. Prognostic value of body mass index before treatment for laryngeal squamous cell carcinoma. Cancer Biol Med. 2015; 12(4): 394-400, doi: 10.7497/j.issn.2095-3941.2015.0043, indexed in Pubmed: 26779376.

6. Cao Dx, Wu Gh, Zhang Bo, et al. Resting energy expenditure and body composition in patients with newly detected cancer. Clin Nutr. 2010; 29(1): 72-77, doi: 10.1016/j.clnu.2009.07.001, indexed in Pubmed: 19647909

7. Moses AWG, Slater C, Preston T, et al. Reduced total energy expenditure and physical activity in cachectic patients with pancreatic cancer can be modulated by an energy and protein dense oral supplement enriched with n-3 fatty acids. Br J Cancer. 2004; 90(5): 996-1002, doi: 10.1038/ sj.bjc.6601620, indexed in Pubmed: 14997196

8. Senkal M, Zumtobel V, Bauer KH, et al. Outcome and cost-effectiveness of perioperative enteral immunonutrition in patients undergoing elective upper gastrointestinal tract surgery: a prospective randomized study. Arch Surg. 1999; 134(12): 1309-1316, doi: 10.1001/ archsurg.134.12.1309, indexed in Pubmed: 10593328.

9. Jankowski M, Las-Jankowska M, Sousak M, et al. Contemporary enteral and parenteral nutrition before surgery for gastrointestinal cancers: a literature review. World J Surg Oncol. 2018; 16(1): 94, doi: 10.1186/ s12957-018-1393-7, indexed in Pubmed: 29769085.

10. Mauskopf JA, Candrilli SD, Chevrou-Séverac H, et al. Immunonutrition for patients undergoing elective surgery for gastrointestinal cancer: impact on hospital costs. World J Surg Oncol. 2012; 10: 136, doi: 10.1186/1477-7819-10-136, indexed in Pubmed: 22770421.

11. Langius JAE, Zandbergen MC, Eerenstein SEJ, et al. Effect of nutritional interventions on nutritional status, quality of life and mortality in patients with head and neck cancer receiving (chemo)radiotherapy: a systematic review. Clin Nutr. 2013; 32(5): 671-678, doi: 10.1016/j. clnu.2013.06.012, indexed in Pubmed: 23845384.

12. Paccagnella A, Morassutti I, Rosti G. Nutritional intervention for improving treatment tolerance in cancer patients. Curr Opin Oncol. 2011; 23(4): 322-330, doi: 10.1097/CCO.0b013e3283479c66, indexed in Pubmed: 21552123.

13. Lu Z, Yang Li, Yu J, et al. Change of body weight and macrophage inhibitory cytokine-1 during chemotherapy in advanced gastric cancer: what is their clinical significance? PLoS One. 2014; 9(2): e88553, doi: 10.1371/ journal.pone.0088553, indexed in Pubmed: 24586342.
14. Martin L, Senesse P, Gioulbasanis I, et al. Diagnostic criteria for the classification of cancer-associated weight loss. J Clin Oncol. 2015; 33(1): 90-99, doi: 10.1200/JCO.2014.56.1894, indexed in Pubmed: 25422490.

15. Rimar KJ, Glaser AP, Kundu S, et al. Changes in Lean Muscle Mass Associated with Neoadjuvant Platinum-Based Chemotherapy in $\mathrm{Pa}$ tients with Muscle Invasive Bladder Cancer. Bladder Cancer. 2018; 4(4): 411-418, doi: 10.3233/BLC-180188, indexed in Pubmed: 30417052.

16. de Las Peñas R, Majem M, Perez-Altozano J, et al. SEOM clinical quidelines on nutrition in cancer patients (2018). Clin Transl Oncol. 2019; 21(1): 87-93, doi: 10.1007/s12094-018-02009-3, indexed in Pubmed: 30617923.

17. Fearon K, Strasser F, Anker S, et al. Definition and classification of cancer cachexia: an international consensus. Lancet Oncol. 2011; 12(5): 489-495, doi: 10.1016/s1470-2045(10)70218-7.

18. Penet MF, Bhujwalla ZM. Cancer cachexia, recent advances, and future directions. Cancer J. 2015; 21(2): 117-122, doi: 10.1097/ PPO.0000000000000100, indexed in Pubmed: 25815852.

19. Castillo-Martinez $L$, et al. Nutritional assesment tools for the identification of malnutrition and nutritional risk associated with cancer treatment. rev Inves Clin. 2018; 70: 121-125.

20. Prado CM, Purcell SA, Laviano A. Nutrition interventions to treat low muscle mass in cancer. J Cachexia Sarcopenia Muscle. 2020; 11(2): 366-380, doi: 10.1002/jcsm.12525, indexed in Pubmed: 31916411.

21. Ravasco P. Nutrition in Cancer Patients. J Clin Med. 2019; 8(8), doi: 10.3390/jcm8081211, indexed in Pubmed: 31416154.

22. Arends J, Bachmann P, Baracos V, et al. ESPEN guidelines on nutrition in cancer patients. Clin Nutr. 2017; 36(1): 11-48, doi: 10.1016/j. clnu.2016.07.015, indexed in Pubmed: 27637832

23. Blum D, Stene GB, Solheim TS, et al. Euro-Impact. Validation of the Consensus-Definition for Cancer Cachexia and evaluation of a classification model--a study based on data from an international multicentre project (EPCRC-CSA). Ann Oncol. 2014; 25(8): 1635-1642, doi: 10.1093/annonc/ mdu086, indexed in Pubmed: 24562443

24. Mantovani G, Macciò A, Bianchi A, et al. Megestrol acetate in neoplastic anorexia/cachexia: clinical evaluation and comparison with cytokine levels in patients with head and neck carcinoma treated with neoadjuvant chemotherapy. Int J Clin Lab Res. 1995; 25(3): 135-141, doi: 10.1007/BF02592554, indexed in Pubmed: 8562975.

25. Silver HJ, Dietrich MS, Murphy BA. Changes in body mass, energy balance, physical function, and inflammatory state in patients with locally advanced head and neck cancer treated with concurrent chemoradiation after low-dose induction chemotherapy. Head Neck. 2007; 29(10): 893-900, doi: 10.1002/hed.20607, indexed in Pubmed: 17405169.

26. Williams JD, Wischmeyer PE. Assessment of perioperative nutrition practices and attitudes-A national survey of colorectal and Gl surgical oncology programs. Am J Surg. 2017; 213(6): 1010-1018, doi: 10.1016/j. amjsurg.2016.10.008, indexed in Pubmed: 27889271.

27. Sriram K, Sulo S, VanDerBosch G, et al. A Comprehensive Nutrition-Focused Quality Improvement Program Reduces 30-Day Readmissions and Length of Stay in Hospitalized Patients. JPEN J Parenter Enteral Nutr. 2017; 41(3): 384-391, doi: 10.1177/0148607116681468, indexed in Pubmed: 27923890.

28. Wischmeyer PE, Carli F, Evans DC, et al. Perioperative Quality Initiative (POQI) 2 Workgroup. American Society for Enhanced Recovery and Perioperative Quality Initiative Joint Consensus Statement on Nutrition Screening and Therapy Within a Surgical Enhanced Recovery Pathway. Anesth Analg. 2018; 126(6): 1883-1895, doi: 10.1213/ ANE.0000000000002743, indexed in Pubmed: 29369092

29. Greco M, Capretti G, Beretta L, et al. Enhanced recovery program in colorectal surgery: a meta-analysis of randomized controlled trials. World J Surg. 2014; 38(6): 1531-1541, doi: 10.1007/s00268-013-2416-8, indexed in Pubmed: 24368573.

30. Gustafsson UO, Oppelstrup $\mathrm{H}$, Thorell A, et al. Adherence to the ERAS protocol is Associated with 5-Year Survival After Colorectal Cancer Surgery: A Retrospective Cohort Study. World J Surg. 2016; 40(7): 1741-1747, doi: 10.1007/s00268-016-3460-y, indexed in Pubmed: 26913728.

31. Kłęk S, Jankowski M, Kruszewski WJ, et al. Clinical nutrition in oncology: Polish recommendations. Nowotwory. Journal of Oncology. 2015; 11(4): 173-190, doi: 10.5603/NJO.2015.0062.

32. Schuetz P, Fehr R, Baechli V, et al. Individualised nutritional support in medical inpatients at nutritional risk: a randomised clinical trial. Lancet. 2019; 393(10188): 2312-2321, doi: 10.1016/S0140-6736(18)32776-4, indexed in Pubmed: 31030981. 